\title{
Service properties of composite electrochemical coating
}

\author{
E. M. Yudina ${ }^{1 *}$, Yu. E. Kisel ${ }^{2}, M . R$. Kadyrov $^{1}$, and A. S. Serguntsov ${ }^{1}$ \\ ${ }^{1}$ Kuban State Agrarian University named after I.T. Trubilin, 13, Kalinina st., Krasnodar, 350044, \\ Russia \\ ${ }^{2}$ Bryansk SAU, 2a, Sovetskaya st., co. Kokino, Vigonichskiy dist., Bryansk reg., 243365, Russia
}

\begin{abstract}
The article considers the ways of solving problems, regarding the rework and durability improvement of elements of industrial equipment. One of the ways to restore and improve the operational properties of machinery parts is the application of galvanic coatings, in particular, composite galvanic coatings. The article shows ways to improve the performance properties of composites, determines the optimal content of the dispersed phase in the composite, in terms of the ratio of the strength of the dispersed phase and matrix. The composite electrochemical coating wear resistance and the roughness of composites under conditions of abrasive wear have been explored. The relationship between the wear resistance and the surface roughness during wear has been determined. In an experimental study of the composite coatings abrasive wear resistance, it was found that their wear largely depends on the size and volume content of dispersed particles in the coating. It has been experimentally proven that the composite coating roughness depends on the matrix mechanical properties, the content and size of disperse particles.
\end{abstract}

\section{Introduction}

The problem of worn-out parts rework and their durability improvement, as much as of units and aggregates of equipment in use under conditions of abrasion wear, is utterly important.

Among the various methods of restoring these parts, it is necessary to choose technological processes that do not change the part microstructure and increase its strength characteristics. The main task of these processes is to protect the restorable parts from corrosion. The restoration of modern technical equipment parts after their depreciation is much cheaper than purchasing and using new spare parts. One of the methods of restoring worn parts is the metal electrolytic deposition (iron-plating, chrome-plating, etc.) on the restored surfaces. Electrolytic iron-plating makes it possible to restore parts of various sizes, shapes, with a wear rate of up to $3,0 \mathrm{~mm}$. The electrodeposition process takes little time, its implementation does not require expensive materials, it can be performed both in the conditions of specialized repair facilities, and in the conditions of workshops of agricultural enterprises. Despite the positive aspects, iron-plating has a number of

*Corresponding author: elena_yudina1963@mail.ru 
disadvantages, preventing its wider spread. These disadvantages are associated with the performance properties of electroplated iron coatings, such as wear resistance, fatigue strength, and base material adhesion strength (the main material of the part). Therefore, under high loads associated with the deformation of the part, it can collapse. In this regard, it is impractical to use pure iron coatings. The introduction of a solid filler (metal oxides, carbides and borides) into the iron matrix, as well as the creation of heterogeneous composite materials consisting of an elastic matrix (iron, nickel, copper) and a solid filler, can significantly increase the operational properties of these coatings.

The wear resistance of dispersion-hardened heterogeneous composite materials can be explained by the fact that strong particles of inclusions, appearing on the surface during the wearing and being contact areas, are subjected to the most intense loading. The high strength of the filler particles prevents wearing of the most loaded protruding areas of the microrelief of the part working surface, providing maximum resistance to plastic deformation during scratching, cutting, compression and surface crushing, which occur during friction [1-3]. Thus, the main functional purpose of filler particles (DP) in a composite is to perceive the loading and distribute it evenly in the binder, and the matrix is to keep the particles from chipping.

The known kinds of composite materials are iron-based composite electrochemical coatings (CEC), which are widely studied and spread, this allows them to be applied to study the relationship between the wear resistance and the strength properties of components [3 - 8].

Therefore, the purpose of the work is to reveal the influence of the component parameters of dispersion-hardened heterogeneous materials on their wear resistance under abrasive wear using the example of CEC.

\section{Materials and methods}

The objects of the research are samples with CEC based on iron alloys with the inclusion of white electrocorundum DP (of M14, M20 grades). The effect of strength properties of coatings on their wear resistance has been studied using the example of CEC iron-nickelelectrocorundum (as less durable) and iron-cobalt-electrocorundum (as more durable), the detailed production technology of which has been described earlier in [5]. Studies of abrasive wear resistance have been carried out according to the GOST 23.208-79 on a specially designed installation. The profile diagram of the worn surface of the coatings has been obtained on a "MarSurf PS1" device. Experimental data have been processed by methods of mathematical statistics [7].

\section{Results and discussions.}

To build a model of the relationship between the wear resistance of CECs with their structure, strength of components and interfacial bonds, we have used the known correlations determining the composition shear strength according to the dislocation theories, constraint equations for normal and tangential stresses, equations for calculating the strength of filler particles for destruction and separation $[8,9]$. The matrix or particle cracking (the strength and structure of which depend on their nature and size) and the violation of the interface between them have been taken as the main mechanisms of the composition destruction $[8,9]$.

The particle strength and $\left(\sigma_{\mathrm{p}}\right)$ and the matrix material strength $\left(\sigma_{m}\right)$, according to the Griffith-Orowan relation [6, 9], can be determined as: 


$$
\begin{gathered}
\sigma_{p}^{2}=\frac{8 \gamma_{p} G_{p}}{\left(1-\mu_{p}\right) d}, \\
\sigma_{m}^{2}=\frac{8 \gamma_{m} G_{m}}{\left(1-\mu_{m}\right) l},
\end{gathered}
$$

where $\gamma_{p}$ and $\gamma_{m}$ - the particles and matrix specific crushing energy, respectively; $G_{p}$ and $G_{m}$ - the particle and matrix shear modulus, respectively; $d$ - the particles equivalent diameter; $l$ - the average distance between the particles; $\mu_{p}$ and $\mu_{m}$ - the particle and matrix Poisson ratio, respectively.

According to the discrete and continuous theories of strength, the average shear stress at fracture $(\tau)$ can be determined using the expressions [7]:

- for one-sided dislocation pileups:

$$
\tau=\frac{G b}{\pi(1-\mu) d},
$$

- for two-sided dislocation pileups:

$$
\tau=\frac{G b}{(1-\mu) d},
$$

where $G$ and $\mu$ - the shear modulus and Poisson ratio of the material, respectively; $b$ - the Burgers vector.

The bond between the disruptive strength and the strength according to the GriffithOrowan for DF particles, can be determined from the expression [7], depending on their size:

$$
\sigma_{p}=\alpha_{p} \tau_{p}
$$

where $\alpha_{p}$ - the ratio, depending on the type of packing of DP particles in the CEC; $\tau_{p}-$ shear stress in DP.

Substituting the equation (5) into the expression (3), considering that, in the case of DP particles brittle fracture, the value is

$$
\gamma_{p}=G_{p} b_{p} / C_{p},
$$

where $G_{p}$ и $b_{p}$ - the shear modulus and Burgers vector, respectively; $C_{p}$ - the constant, depending on the DP material features, let us define particle strength:

$$
\sigma_{p}=\alpha_{p} G_{p} / C_{p}
$$

Let us define the greatest strength of non-destructible DP particles: for crack growth within one grain $\left(d_{p d}\right)$ according to the discrete theory

$$
d_{p d}=\left(2 / \alpha_{p}^{2}\right) b_{p} C_{p} /(1-\mu)
$$

with an elliptical crack form $\left(d^{e}{ }_{p c}\right)$ according to the continuum theory

$$
d_{p c}^{e}=\left(4 / \alpha_{p}^{2}\right) \quad b_{p} C_{p} /(1-\mu),
$$

and a cylindrical crack form $\left(d^{c}{ }_{p c}\right)$

$$
d_{p c}^{c}=\left(8 / \pi \alpha^{2}\right) \quad b_{p} C_{p} /(1-\mu) .
$$


The composition highest strength will be achieved at the distance between the DP particles under the next condition:

$$
\tau_{\kappa}=\tau_{p},
$$

where $\tau_{\kappa}$ and $\tau_{p}$ - the theoretical shear strength of the composite and DP particles, respectively.

Substituting the values of quantities for the discrete theory, we obtain the critical distance between particles $\left(\lambda_{d}\right)$ :

$$
\lambda_{d}=1 /(1-\mu)\left(G_{m} b_{m} C_{p}\right) /\left(\pi G_{p}\right) .
$$

Changing the expression $\gamma_{m}=G_{m} b_{m} / C_{m}$ и $C_{m} \approx C_{p}$ - for the matrix, the expression (12) takes the next form:

$$
\lambda_{d}=1 /(1-\mu)\left(\gamma_{m} / \gamma_{p}\right) b_{p} C_{m} / \pi
$$

According to the continuum strength theory, the expression for the distribution of the critical distance between particles $\left(\lambda_{c}\right)$ :

$$
\lambda_{c}=1 /(1-\mu)\left(\gamma_{m} / \gamma_{p}\right) b_{p} C_{p} .
$$

Since the composite maximum strength cannot exceed the particles strength, the ratio $\sigma_{p} / \sigma_{\kappa}=1$ should be valid for the most durable composite. Then the optimal content of DP in the CEC, which corresponds to the maximum strength of the material during its destruction (the crack passes through the matrix and dispersed particles), can be determined from the condition:

$$
\sigma_{p} / \sigma_{\kappa}=K_{p}=\beta \lambda_{\kappa} / d_{\kappa} \text { or } \sigma_{p}^{2} / \sigma^{2}=K_{p}^{2}=\left(\beta \lambda_{\kappa} / d_{\kappa}\right)^{2},
$$

where $\beta=\lambda_{i} / d_{i} ; \quad d_{i}$ и $\lambda_{i}-$ the largest size of non-destructible particles and the critical distance between them.

Let us consider the case of the fracture of composition by the example of calculating the strength according to the discrete theory of dislocations and the passage of cracks inside one grain. For such a case:

$$
\sigma_{p}^{2} / \sigma^{2}{ }_{\kappa}=\frac{\frac{2 \gamma_{p} G_{p}}{(1-\mu) d_{\kappa}}}{\alpha_{p}^{2} \frac{G_{m} b_{m} G_{p}}{\pi(1-\mu) \lambda C_{p}}}=\frac{2 \pi}{\alpha_{p}^{2}} \frac{\lambda}{d_{p}} \frac{\gamma_{p}}{\gamma_{m}}=\frac{4 \lambda_{\kappa}}{\left(\pi d_{k}\right)^{2}},
$$

The expression (16) allows determining the ratio $\lambda / d$ for this case in the next form:

$$
\frac{\lambda}{d_{p}}=\frac{\pi^{3}}{8 \alpha_{p}^{2}} \frac{\gamma_{p}}{\gamma_{M}}
$$

According to [6], the relationship (17) with the content of the dispersed phase in the composite can be expressed by the next ratio:

$$
\frac{\lambda}{d_{p}}=\alpha \sqrt{\frac{\pi}{3 V_{d p}}}-\sqrt{\frac{2}{3}},
$$

which is connected with the CEC component strength properties:

$$
\frac{\lambda}{d_{p}}=\frac{\pi^{3}}{8 \alpha_{p}^{2}} \frac{\gamma_{p}}{\gamma_{m}}=\sqrt{\frac{\pi}{3 V_{d p}}}-\sqrt{\frac{2}{3}} .
$$


From the expression (19), we obtain the dispersed phase volumetric content, which corresponds to the composite highest strength within the discrete theory:

$$
V_{d p}^{d}=\frac{\pi}{3} \frac{1}{\left(\frac{\pi^{3}}{8 \alpha_{p}^{2}} \frac{\gamma_{p}}{\gamma_{m}}+\sqrt{\frac{2}{3}}\right)^{2}},
$$

Similarly, within the continuum theory:

$$
V_{d p}^{c}=\frac{\pi}{3} \frac{1}{\left(\frac{\pi^{3}}{\alpha_{p}^{2}} \frac{\gamma_{p}}{\gamma_{m}}+\sqrt{\frac{2}{3}}\right)^{2}} .
$$

In the case of a two-sided accumulation of dislocations for elliptical and cylindrical cracks, we, accordingly, obtain:

$$
\begin{aligned}
V_{d p}^{c e} & =\frac{\pi}{3} \frac{1}{\left(\frac{1}{\alpha_{p}^{2}} \frac{\gamma_{p}}{\gamma_{m}}+\sqrt{\frac{2}{3}}\right)^{2}}, \\
V_{d p}^{c c} & =\frac{\pi}{3} \frac{1}{\left(\frac{8}{\alpha_{p}^{2}} \frac{\gamma_{p}}{\gamma_{m}}+\sqrt{\frac{2}{3}}\right)^{2}} .
\end{aligned}
$$

The expressions (20) - (23) have been obtained under the condition of the presence of an "ideal" connection between the CEC components at the level of the highest matrix strength, which has been taken for an electrolytic iron $\gamma_{m}{ }^{i}=2000 \mathrm{erg} / \mathrm{cm}^{2}$, a copper $\gamma_{m}{ }^{c}=$ $1200 \mathrm{erg} / \mathrm{cm}^{2}$, a nickel $\gamma_{m}{ }^{n}=1700 \mathrm{erg} / \mathrm{cm}^{2}$ [5]. Its actual value can be in the range of $0 \leq$ $\sigma_{\mathrm{p}-\mathrm{m}} \leq \sigma_{\mathrm{p}}$.

Verification of the calculations, using the example of composite coatings "ironelectrocorundum", "nickel-electrocorundum" and "copper-electrocorundum" [4, 5], showed that the results of calculations using the equation (22), based on the continuum theory (cracks are modeled as two-sided accumulations), are more accurate. The calculated content of solid DP in wear-resistant iron-based CECs, should be 25 ... 27\% (vol.), copper-based 8 ... 10\% (vol), nickel-based - $18 \ldots 20 \%$ (vol.).

Thus, the composite wear resistance should increase with an increase in the strength of the matrix and the bond strength of the dispersed phase and the matrix. The strength of "pure" electrolytic iron can be increased by introducing alloying elements of cobalt, nickel and others into the solution. The deposited alloys have higher elastic-plastic properties, wear resistance and adhesion strength to the base, and lower internal stresses [5].

The experimental studies of the abrasive wear resistance of CECs have qualitatively confirmed the theoretical calculations. The wear resistance of composites in contact with an abrasive largely depends on the size and volume content of dispersed particles in the coating. The wear of the CEC based on iron-nickel and iron-cobalt alloys, with an increase in the content of the dispersed phase in the coating, has decreased and has passed through a minimum at a content of micropowders ( $V$ ) of the order of $18 \ldots 26 \%$ (vol.).

At the same time, the CEC wear resistance, based on iron-cobalt coatings $(V=24 \ldots$ $26 \%$ (vol.)) has been higher than that of composites, based on iron-nickel coatings ( $V=18$ ... 22\% (vol.)), which, probably, due to the greater strength of the Fe-Co binder. Moreover, with an increase in the DP content higher the optimal one, the CEC wear has increased insignificantly while maintaining the established nature of the process.

The study of the samples surface roughness, formed in the process of wear tests, showed that with an increase in the volume content of the dispersed phase in composite 
coatings from 0 to 5 ... 7\% (vol.), their roughness has slightly increased (Fig. 1). With a further increase in the content of the dispersed phase in the composite (for up to 18 ... $25 \%$ (vol.)), the roughness has decreased (to $R a 0.5 \ldots 0.7 \mu \mathrm{m}$ ), reaching a minimum in the content of particles in the coating that corresponds to the highest wear resistance of the heterogeneous material, and then has increased relatively sharply. Furthermore, the weaker and the softer Fe-Ni-based CECs have had higher roughness than Fe-Co based CECs. It should be noted that the connection, we have sighted, between the material wear resistance and the roughness of the contact surface with the mating part, formed during friction, is also noted in the literature for other materials [2]. The relationship between wear resistance and roughness of working surfaces is explained as follows. When testing coatings with no DP content, the unevenness of their contact surface with the counterface is determined by the anisotropy of the structure and elastic-plastic properties of crystals. Therefore, the formed roughness of the working surface of iron-cobalt coatings is somewhat higher than that of iron-nickel ones.

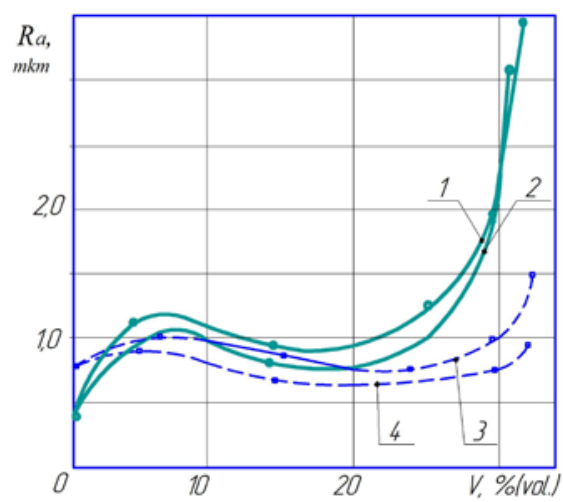

1 - Fe-Ni-Al ${ }_{2} \mathrm{O}_{3}$ (M14); 2 - Fe-Ni-Al $2 \mathrm{O}_{3}$ (M20); 3 - Fe-Co- $\mathrm{Al}_{2} \mathrm{O}_{3}$ (M14); 4 - Fe-Co-Al $\mathrm{O}_{3}$ (M20)

Fig. 1. The dependence of the roughness of composite electrochemical coatings on the content $(V, \%$ (vol.)) and the sizes of the dispersed phase (M14, M20) in the coating

With the appearance and increase of the DP content in the coatings, its particles replace the most loaded protruding areas of microroughness of the working surface relief, providing maximum resistance to plastic deformation during friction. The protrusions of the working surface can be modeled as a set of hemispheres (Fig. 2, a). With an increase in the content of DP in the CEC up to $5 \ldots 7 \%$ (vol.), the replacement process occurs very actively, which noticeably increases the coating wear resistance and reduces its roughness.

At the initial stage of the part working surface "filling" process with DP particles, they undergo a large contact load from the roughness of the counterface. Due to its limited connection with the matrix and strength, there is a noticeable chipping of certain embedded particles with a roughening of the CEC surface (Fig. 2, b).

For this reason, the less strong iron-nickel bond retains DP particles worse and coarsens more than the iron-cobalt one (Fig. 1). 


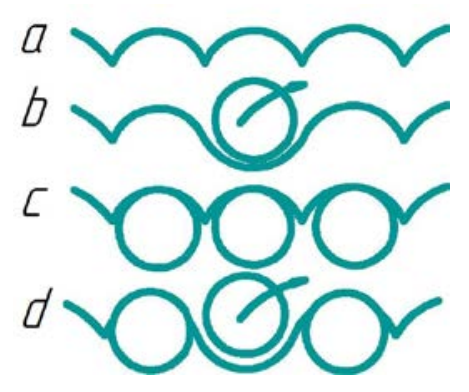

Fig. 2. The model of a rough surface of "pure" coatings (a), coatings with pre- optimal (b), optimal (c) and higher than optimal (d) content of DP particles

With an increase in the DP content in the CEC for up to $15 \ldots 22 \%$ (vol.), the density of the surface protrusions, replaced by solid embedded particles, has increased, leading to a corresponding increase in the strength of the heterogeneous material and to a decrease in the roughness of its working surface (Fig. 2, c). Due to the redistribution of the load between the particles, they have been better retained by the bundle, increasing the CEC fraction resistance ability. It is interesting that in the region of the highest strength of a particular composite material and its optimal (best) wear resistance, the formed roughness of various CECs has similar values depending on their operating conditions (see Fig. 1 and 2, c).

With an increase in the DP content in the CEC of a more optimal one and a decrease in the ability of the binder to hold DP particles, their chipping increases again and the roughness of the composite working surface increases rather sharply (Fig. 2, d).

Thus, in the absence of DP particles and with a low content of them in the CEC, the formed roughness of the composite working surface in contact with the counterface quite significantly depends on the strength and ability to self-hardening of the coating material (matrix or binder). In the area of optimal highest wear resistance of CECs, their roughness depends on the physical-mechanical properties of the filler and the ability of the binder to retain DP particles.

The studies conducted, made it possible to recommend composite coatings for the restoration of parts subject to abrasive wearing $[10,11]$.

\section{Conclusion}

The relationship between the parameters of the composite materials heterogeneous structure and their strength properties with wear resistance and formed roughness in the process of abrasive wearing has been theoretically and experimentally established. It has been found that the highest wear resistance of a composite under conditions of abrasive wearing is determined by its strength, which depends on the strength of the components and can be calculated using the continuum theory of dislocations. The most wear-resistant CECs under abrasive wearing conditions have a formed roughness of the same order, determined by the friction conditions and strength properties of the composites.

\section{References}

1. Kragelskiy I V, Mikhin N M 1984 Machine friction units: Handbook.

2. Ainbinder S B et al. 1981 Roughness parameters of the counterface, determining the wear resistance of polyethylene Friction and wear. 2 (1) 12-21. 
3. Guryanov G V et al 2009 Application of the finite-element method for calculating the elastic characteristics of composite materials Proceedings of the Kuban State Agrarian University 4 (19) 235-239.

4. Petrov Yu N , Guryanov G V , Bobanova Zh I 1990 Electrolytic iron deposition Shtiintsa

5. Yu.E. Kisel et al 2020 J. Phys.: Conf. Ser. 1515052018

6. Martin D U et al. 1983 Micromechanisms of dispersion hardening of alloys Metallurgia

7. Friedel Sh. Dislocations 1967 Mir

8. Cotrell A 1969 The theory of dislocations Mir

9. Ivanova V S 1965 The role of dislocations in the strengthening and destruction of metals Nauka

10. E.M. Yudina et al 2021 IOP Conf. Ser.: Mater. Sci. Eng. 1064012044

11. E M Yudina, G V Guryanov, V A Popov 1994 Abrasive wear resistance for composite electroplates Elektronnaya Obrabotka Materialov 1 38-41 